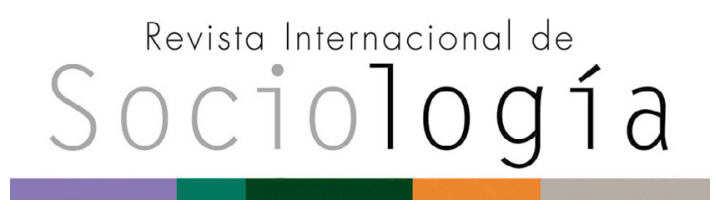

Revista Internacional de Sociología RIS

vol. 79 (1), e179, enero-marzo, 2021, ISSN-L:0034-9712 https://doi.org/10.3989/ris.2021.79.1.19.135

\section{TIPOLOGÍAS BASADAS EN LA ADHESIÓN A LOS MITOS SOBRE LA VIOLENCIA DE GÉNERO: EVIDENCIAS DE UN ANÁLISIS DE CLASES LATENTES}

\author{
CARMEn M. a LeÓN \\ Universidad de Castilla-La Mancha \\ Carmen.Leon@uclm.es \\ ORCID iD: https://orcid.org/0000-0002-0591-6056 \\ Eva AizPURÚA \\ City, University of London y Trinity College Dublin \\ Eva.Aizpurua@city.ac.uk \\ ORCID iD: https://orcid.org/0000-0001-7045-5535
}

Cómo citar este artículo / Citation: Carmen M. ${ }^{a}$ León y Eva Aizpurúa. 2021. "Tipologías basadas en la adhesión a los mitos sobre la violencia de género: Evidencias de un análisis de clases latentes". Revista Internacional de Sociología 79(1):e176. https://doi.org/10.3989/ ris.2021.79.1.19.135

\begin{abstract}
Resumen
Los mitos sobre la violencia de género favorecen la persistencia del maltrato al actuar como elementos justificativos de las agresiones. El presente trabajo contribuye a la escasa evidencia acumulada al emplear un análisis de clases latentes (ACL) para estudiar tipologías de personas basadas en la adhesión a diez mitos y creencias sobre la violencia de género y examinar las características que definen a cada una de las clases. Para ello, se contó con una muestra compuesta por 1.007 personas $(51,1 \%$ mujeres y 48,9 \% hombres; $M=45,37$ años). Los resultados muestran un patrón de tres clases latentes: adhesión baja ( $n=663 ; 65,8 \%$ ), adhesión moderada ( $n=113 ; 11,2 \%)$ y adhesión alta ( $n=231 ; 22,9 \%)$. Los grupos presentan diferencias cuantitativas y cualitativas en su composición y la pertenencia a las clases con mayor aceptación de mitos se relaciona con actitudes más sexistas.
\end{abstract}

\section{Palabras clave}

Violencia de género, mitos, análisis de clases latentes, tipologías, sexismo.

\section{TYPOLOGIES BASED ON ADHER- ENCE TO MYTHS ABOUT INTIMATE PARTNER VIOLENCE AGAINST WOMEN: EVIDENCE FROM A LA- TENT CLASS ANALYSIS}

Copyright: (C) 2021 CSIC. Este es un artículo de acceso abierto distribuido bajo los términos de la licencia de uso y distribución Creative Commons Reconocimiento 4.0 Internacional (CC BY 4.0).

Recibido: 14/10/19. Aceptado: 21/07/20.

Publicado: 06/04/2021

\section{Abstract}

Myths about intimate partner violence against women favour abuse by functioning as elements justifying aggressions. The current research contributes to the limited research in this area by using latent class analysis (LCA) to identify typologies based on adherence to ten myths and beliefs about intimate partner violence and then examining the characteristics defining each of the classes. For this, a sample composed of 1,007 individuals $(51.1 \%$ women and $48.9 \%$ men; $M=45.37$ years) was used. The results reveal a pattern of three latent classes: low adhesion $(n=663$; $65.8 \%)$, moderate adhesion $(n=113 ; 11.2 \%)$, and high adhesion ( $n=231 ; 22.9 \%)$. The classes differed quantitatively and qualitatively and belonging to classes featuring a greater acceptance of these myths was associated with more sexist attitudes.

\section{KeYWORDS}

Intimate partner violence, myths, latent class analysis, typologies, sexism. 


\section{INTRODUCCIÓN}

La violencia de género, entendida como aquella que se ejerce sobre las mujeres por parte de sus parejas o exparejas, es uno de los problemas sociales y de salud pública más graves que sufren las mujeres en el mundo (Organización Mundial de la Salud 2017). Además de sus repercusiones en el bienestar y la salud de las víctimas (Coker et al. 2000; Domenech del Río y Sirvent García del Valle 2016), la violencia de género supone una violación de los derechos humanos, constituyendo la máxima expresión de la desigualdad entre mujeres y hombres. Estimaciones mundiales muestran que tres de cada diez mujeres de 15 o más años han sufrido violencia física o sexual en algún momento de sus vidas por parte de sus parejas o exparejas (Organización Mundial de la Salud 2017). A nivel nacional, la Macroencuesta de Violencia contra la Mujer concluye que una de cada tres mujeres $(32,4 \%)$ de 16 o más años residentes en España ha sufrido alguna forma de violencia en la pareja a lo largo de su vida (Delegación del Gobierno contra la Violencia de Género 2020).

A pesar de los esfuerzos para erradicar esta forma de violencia, las estadísticas continúan arrojando cifras preocupantes. En una conocida revisión, Flood y Pease (2009) destacaron la importancia de ciertas creencias en la persistencia de la violencia de género, al actuar como elementos justificativos del maltrato. Como la literatura ha apuntado, si la sociedad percibe que estos comportamientos son aceptables, se generará más violencia; si la ciudadanía tolera y/o justifica tales acciones, será más probable que estas conductas perduren y se reproduzcan, ya que prolifera un clima social de tolerancia donde se condona el comportamiento de los maltratadores y se dificulta que las mujeres abandonen relaciones abusivas, denuncien la violencia sufrida y cuenten con el apoyo que precisan (Copp, Giordano, Longmore y Manning 2016; Ferrer y Bosch 2014; Gracia, Lila y Santirso 2020).

En este punto entran en juego los mitos sobre la violencia de género, es decir, aquellas creencias fundamentadas en estereotipos sobre esta forma de violencia que, aun siendo falsas, se encuentran ampliamente arraigadas y son a menudo utilizadas para minimizar, justificar o negar la agresión a la pareja (Peters 2008). Al contrario de lo que ocurre con los mitos de la violación, la literatura que ha abordado el estudio de los mitos sobre la violencia de género es limitada (Megías, Toro-García y Carretero-Dios 2018), lo que pone de relieve la conveniencia de desarrollar investigaciones que prueben la falsedad de estos mitos y detengan sus efectos nocivos (Bosch y Ferrer 2012; Grothues y Marmion 2006).

\section{Antecedentes teóricos y empíricos}

El estudio de los mitos sobre la violencia de género encuentra sus antecedentes en las investigaciones sobre los mitos de la violación (para una revisión amplia, véase Bohner et al. 2009 y Megías et al. 2011). Estos trabajos han puesto de relieve la importancia de los mitos como elementos a través de los cuales se responsabiliza a las víctimas, se exime de responsabilidad a los agresores, se minimizan las consecuencias negativas de la violación y se caracterizan de forma distorsionada y estereotípica los sucesos (Toro 2018). Además, estos estudios apuntan a la eficacia de los mitos como elementos justificativos de las agresiones, ya que una parte de ellos aluden a ideas del tipo "ella podría haber evitado el suceso, probablemente lo ha provocado o inconscientemente lo deseaba" (Peters 2008: 3).

Más recientemente, se han extraído conclusiones similares en el ámbito de la violencia de género (Bosch y Ferrer 2002; 2012; Bosch et al. 2013; Leung 2019; Megías, Toro-García y Carretero-Dios 2018; Policastro y Payne 2013; Yamawaki et al. 2012; Westbrook 2009). Uno de los trabajos más relevantes a nivel nacional fue el desarrollado por Bosch y Ferrer (2012), quienes propusieron una clasificación de los principales mitos sobre la violencia de género en cinco categorías, diferenciando entre los mitos sobre la marginalidad del fenómeno, los referidos a los maltratadores, los mitos sobre las mujeres maltratadas, aquellos que minimizan la importancia de la violencia de género y los mitos negacionistas.

Los mitos sobre la marginalidad sitúan la violencia de género en el terreno de la excepcionalidad, manteniéndola como algo alejado del propio grupo y fruto de circunstancias particulares (Bosch y Ferrer 2012). Algunas de las justificaciones más habituales son afirmar que la violencia de género solo ocurre en países subdesarrollados o en hogares con problemas socioeconómicos (p. ej., entre familias o personas con escasos recursos). Los mitos sobre los maltratadores ponen el acento en ciertos factores personales del agresor que le habrían conducido hasta la violencia y que, de un modo más o menos explícito, lo exoneran de responsabilidad (Bosch y Ferrer 2012). Algunas de las creencias más habituales mantienen que los maltratadores han sido, a su vez, personas maltratadas en su infancia, son enfermos mentales o tienen problemas con el consumo de alcohol u otras drogas.

Por su parte, los mitos sobre las mujeres maltratadas responsabilizan a estas de lo que les sucede, bien porque algunas de sus características "atraen la violencia" o bien porque consienten o solicitan esa violencia (Bosch y Ferrer 2012). Entre las justificaciones más habituales se encuentran aquellas que hacen referencia al estereotipo de mujer que activamente provoca la violencia que sufre; creencias sobre la naturaleza masoquista y sumisa de las mujeres que no ponen fin al abuso y permanecen con el agresor, y las ideas sobre las supuestas ca- 
racterísticas de las mujeres maltratadas (mujeres pasivas, sumisas, con baja autoestima, sin trabajo remunerado, etc.).

Los mitos que minimizan la importancia de la violencia de género cuestionan la gravedad y la existencia misma del fenómeno (Peters 2008). Entre los más destacados se encuentran aquellos que sostienen que la violencia de género es un fenómeno puntual y muy localizado, los que defienden que la violencia psicológica no es tan grave como la física y los que aseguran que hombres y mujeres son igual de violentos en las relaciones de pareja. Para finalizar, los mitos negacionistas no solo niegan la existencia de la violencia de género, sino que van más allá al considerar que esta forma de violencia es una exageración creada y utilizada por determinadas mujeres para perjudicar a los hombres (Bosch y Ferrer 2012). Algunas de las justificaciones más habituales son afirmar que los hombres son tan victimizados como las mujeres en las relaciones de pareja y que gran parte de las denuncias por violencia de género son falsas.

A pesar del amplio desarrollo teórico, han sido escasos los acercamientos empíricos a la evaluación de los mitos sobre la violencia de género (Briere 1987; Peters 2008; Saunders et al. 1987). En España, uno de los estudios pioneros en abordar esta cuestión fue el realizado por el Centro de Investigaciones Sociológicas sobre la percepción social de la violencia de género (CIS 2012). Esta investigación mostró que el 43,2 \% de las personas encuestadas consideraban que hay más agresores entre los extranjeros que entre los españoles. Asimismo, una proporción similar $(37,6 \%)$ se mostró de acuerdo con que los agresores suelen tener alguna enfermedad mental. Aproximadamente un tercio de las personas encuestadas $(34,6 \%)$ indicaron que si las mujeres sufren maltrato es porque quieren, mientras que un $52,5 \%$ y un $41,9 \%$ afirmaron, respectivamente, que las mujeres extranjeras y aquellas que residen en entornos rurales y municipios pequeños son más vulnerables a convertirse en víctimas de malos tratos. Más recientemente, otro estudio realizado por el CIS sobre percepciones sociales de la violencia sexual en España mostró que casi tres de cada diez personas estuvieron de acuerdo con que muchas mujeres tienden a exagerar el problema de la violencia machista (CIS 2017). A nivel europeo, los datos del último Eurobarómetro sobre violencia de género muestran que un $17,0 \%$ de las personas encuestadas estuvieron de acuerdo con que la violencia de género es a menudo provocada por las mujeres (Comisión Europea 2016).

También es destacable el estudio efectuado por Policastro y Payne (2013), quienes evaluaron la prevalencia de una serie de mitos sobre la violencia de género entre la población universitaria. Además de encontrar un desacuerdo generalizado con los mitos estudiados, se halló que el apoyo a unos mitos se relacionaba con el respaldo a otros. Estos hallazgos proporcionan evidencias de la interdependencia de los mitos también en el plano empírico y no solo a nivel teórico (Ferrer y Bosch 2012).

\section{Predictores de la adhesión a los mitos sobre la violencia de género}

Las evidencias disponibles ponen de manifiesto la importancia de ciertas variables, como el sexo y la ideología sexista, en el respaldo a los mitos sobre la violencia de género. En relación con el sexo, se ha encontrado que, si bien hombres y mujeres suscriben mitos sobre la violencia de género, esta tendencia parece mayor entre ellos (Gracia y Herrero 2006; Megías, Toro-García y Carretero-Dios 2018; Nabors, Dietz y Jasinski 2006; Yamawaki et al. 2012). Como muestra de ello, Policastro y Payne (2013), en su estudio con población universitaria, hallaron que los hombres, en comparación con las mujeres, tenían más probabilidades de apoyar los mitos analizados. Más recientemente, Megías y sus colaboradores (2018) encontraron que los hombres respaldaban, en mayor medida que las mujeres, los mitos sobre la violencia de género en las seis muestras empleadas. No obstante, estos resultados son contrarios a los hallados por Webster y sus colaboradores (2018) en un estudio realizado con una muestra representativa de la población australiana, donde se encontró que las creencias sobre la igualdad de género eran más relevantes que el sexo en la explicación de las actitudes hacia la violencia de género.

En relación con las creencias sexistas, las evidencias muestran que las personas más sexistas suscriben en mayor medida los mitos sobre la violencia de género (Marques-Fagundes et al. 2015; Megías, Toro-García y Carretero-Dios 2018). Por ejemplo, Peters (2008) halló que los mitos sobre la violencia doméstica eran respaldados más fuertemente por los hombres y las mujeres que se adherían a estereotipos y creencias tradicionales sobre los roles de género. En líneas similares, Marques-Fagundes y sus colaboradores (2015), en un estudio realizado con mujeres pertenecientes a distintas organizaciones de mujeres en España, hallaron que las encuestadas con creencias más igualitarias mostraban menor aceptación de los mitos sobre la violencia de género.

Otros hallazgos menos sólidos han relacionado el respaldo a los mitos con otras variables como la edad y el nivel educativo. Con respecto a la edad, las evidencias acumuladas no son consistentes; mientras que algunos estudios no han encontrado que esta variable se relacione con la adhesión a los mitos y creencias sobre la violencia de género (Megías, Toro-García y Carretero-Dios 2018), otros sugieren que estas creencias disminuyen con la edad (Simon 
et al. 2001), o bien que ambas variables presentan una relación curvilínea, de tal forma que las actitudes de apoyo hacia la violencia de género son mayores entre los jóvenes, más bajas en la edad adulta y nuevamente elevadas entre las personas más mayores (Webster et al. 2018).

En relación con la educación, las evidencias muestran que el nivel educativo se relaciona de forma negativa con el respaldo a ciertos mitos (Nabors, Dietz y Jasinski 2006; Policastro y Payne 2013; Webster et al. 2018). Concretamente, Policastro y Payne (2013) encontraron que los años de estudio se asociaban negativamente con los indicadores "me resulta difícil entender por qué las mujeres maltratadas permanecen en relaciones violentas" y "no creo que sea tan difícil para las mujeres abandonar las relaciones violentas". En estos supuestos, las personas que se encontraban en las etapas más tempranas de su carrera universitaria se mostraban más de acuerdo con estos indicadores que aquellas que se hallaban en una etapa más avanzada de sus estudios. En líneas similares, Webster y sus colaboradores (2018) hallaron que las personas con educación secundaria o inferior presentaban mayores actitudes de apoyo hacia la violencia de género en comparación con las que indicaron tener estudios universitarios.

Además, y a pesar de que no se han localizado estudios previos que hayan analizado su efecto, se considera necesario examinar otros factores que podrían modular la adhesión a los mitos sobre la violencia de género, como la ideología política, la religiosidad, la orientación sexual, el nivel de ingresos, el lugar de residencia, el estado civil, el país de origen y la victimización propia y vicaria en el ámbito de la pareja, dada su vinculación con otras esferas íntimamente relacionadas con la violencia de género, como la percepción social sobre el fenómeno y el sexismo (Alfredsson, Ask y Von Borgstede 2016; Dalal, Lee y Gifford 2012; Dardis et al. 2017; Edwards 2014; Gracia, Herrero y Lila 2008; Herrero, Rodríguez y Torres 2017; Waltermaurer 2012; Wang 2016).

\section{Presente estudio}

Los mitos y creencias sobre la violencia de género facilitan la persistencia del maltrato al actuar como elementos justificativos de las agresiones contra las mujeres (Peters 2008). Además de guardar relación con la atribución de responsabilidad a las víctimas y la exoneración de los maltratadores (Megías, Toro-García y Carretero-Dios 2018), el apoyo a los mitos sobre la violencia de género dificulta el desarrollo de intervenciones y reduce la probabilidad de que las víctimas reciban la ayuda que precisan (Grothues y Marmion 2006; Roberts 2002).

Debido a la escasa atención científica que ha recibido este ámbito de estudio, el presente trabajo pretende contribuir a este cuerpo de investigación identificando tipologías basadas en la adhesión a mitos y creencias sobre la violencia de género en España, así como evaluar las características distintivas de cada grupo y su relación con las actitudes sexistas. Para ello, se aplicó un análisis de clases latentes ( $A C L)$, una técnica de interdependencia poco empleada en el ámbito de estudio de la violencia contra las mujeres (vid. Ansara y Hindin 2010; Parker et al. 2016; Saint-Eloy et al. 2017) y de la cual no se tiene constancia de que haya sido aplicada al estudio de los mitos sobre la violencia de género, lo que supone una novedad de esta investigación. Partiendo de la interdependencia de estos mitos y creencias (Bosch y Ferrer 2012; Policastro y Payne 2013), el ACL permite explorar los patrones de adhesión a través de la identificación de clases que más tarde serán utilizadas para examinar su vinculación con la ideología sexista y las experiencias de victimización en el ámbito de las relaciones de pareja. Se espera que los hallazgos de la presente investigación sirvan para orientar futuras intervenciones dirigidas a erradicar falsas creencias sobre la violencia de género.

\section{MÉtodo}

\section{Participantes y procedimiento}

En el estudio han participado 1.007 panelistas $^{1}$ de Netquest residentes en España (48,9 \% hombres y $51,1 \%$ mujeres). Sus edades oscilaron entre los 18 y los 86 años, siendo la media de edad 45 años ( $D T=$ 15,16). La muestra se extrajo mediante muestreo no probabilístico por cuotas de sexo, edad y hábitat. De forma previa a la recogida de los datos, se evaluó la claridad e idoneidad del instrumento. La revisión del cuestionario se realizó mediante un papel de personas expertas. Los datos se recogieron entre el 7 y el 29 de enero de 2019 utilizando una encuesta online cuyo tiempo medio de cumplimentación fue de aproximadamente 12 minutos $(M=11,72 ; D T=6,32)$. El cuestionario fue completado por el $92,3 \%$ de las personas invitadas.

\section{Variables}

Escala de mitos y creencias sobre la violencia de género. La escala fue elaborada ad hoc y estuvo compuesta por diez ítems dirigidos a evaluar una serie de mitos y creencias sobre la violencia de género que pretendían representar las cinco categorías identificadas por Bosch y Ferrer (2012): los mitos sobre la marginalidad, los mitos sobre los maltratadores, los mitos sobre las mujeres maltratadas, los mitos que minimizan la importancia de la violencia de género y los mitos negacionistas (véase la tabla I). Los ítems fueron evaluados con escalas de cinco puntos que oscilaron entre "muy en desacuerdo" y 
"muy de acuerdo", y que posteriormente fueron dicotomizados para distinguir entre quienes estaban de acuerdo con las afirmaciones ("de acuerdo" y "muy de acuerdo") y quienes no (incluyendo "ni de acuerdo ni en desacuerdo", "en desacuerdo" y "muy en desacuerdo"). La consistencia interna de la escala fue satisfactoria, tanto con los ítems originales como tras la dicotomización de los indicadores $\left(\alpha=0,76 ; K R_{20}\right.$ $=0,60)$. Asimismo, el análisis factorial exploratorio realizado sobre la matriz de correlaciones tetracóricas mostró que todos los indicadores saturaron en un único factor que explicó el $72,6 \%$ de la varianza (las cargas factoriales oscilaron entre 0,50 y 0,76 , a excepción del ítem 8, cuya saturación fue de 0,24).

Escala de sexismo clásico y moderno $(\alpha=, 83)$. La escala empleada fue una adaptación de la Swedish Modern and Classical Sexism Scale, desarrollada y validada por Ekehammar, Akrami y Araya (2000) en el contexto escandinavo. Para la elaboración de la escala, los autores se basaron en el término de 'sexismo moderno' propuesto por Swim et al. (1995). La adaptación de la escala consistió en la exclusión de un indicador, tras revelar los resultados del análisis factorial exploratorio realizado sobre la matriz de correlaciones policóricas que uno de los indicadores presentaba una saturación muy baja $(\lambda=, 06)$. Todos los demás se agrupaban en un único factor (eigenvalue $=4,99 ; 85,0 \%$ de varianza explicada), con cargas factoriales superiores a 0,30. La versión empleada está compuesta por 14 indicadores con escalas de respuesta de cinco puntos que oscilan entre "muy en desacuerdo" y "muy de acuerdo". Para minimizar los sesgos de aquiescencia ${ }^{2}$, seis indicadores estaban formulados en dirección opuesta y fueron posteriormente recodificados para que, al calcular la media de los ítems, las puntuaciones más elevadas representaran mayores niveles de sexismo.

Conocer a víctimas de violencia de género. Se pidió a las personas encuestadas que indicaran si, dentro de su entorno cercano, conocían a alguna mujer que hubiese sufrido violencia por parte de su pareja o expareja ("Dentro de tu entorno cercano (círculo de amigos/as, familiares, compañeros/as de trabajo o estudios... ¿conoces a alguna mujer que haya sufrido violencia por parte de su pareja o expareja? Por violencia nos referimos a todos aquellos comportamientos que forman parte del maltrato físico, psicológico y sexual"). Las opciones de respuesta fueron "no", "sí" y "no lo sé". Debido a la sensibilidad del tema se incluyó la opción de respuesta "prefiero no contestar".

Conocer a hombres maltratadores. Posteriormente, se solicitó que indicaran si, dentro de su entorno cercano, conocían a algún hombre que hubiese ejercido violencia contra su pareja o expareja. Nuevamente, las opciones de respuesta fueron "no", "sí", "no lo sé" y "prefiero no contestar".

Victimización propia. Por último, se les pidió que señalaran si, a lo largo de su vida, habían sufrido alguno(s) de los siguientes comportamientos por parte de sus parejas o exparejas: conductas de control (p.ej., impedirle ver a amigos o familiares, insistir en saber dónde está en cada momento, etc.), maltrato verbal (p.ej., amenazas, insultos...), maltrato físico (p.ej., empujones, golpes, etc.) o violencia sexual (p.ej., obligarle a mantener relaciones sexuales sin su consentimiento). Las preguntas fueron de respuesta dicotómica sí/no, incluyendo la opción de respuesta "prefiero no contestar".

Características personales. Como variables de control se incluyeron el sexo, la edad (en intervalos), el nivel educativo (sin estudios o estudios primarios; secundaria, FP y bachillerato; licenciatura, diplomatura o grado; máster o doctorado), la orientación política, evaluada a través de una escala de autoubicación ideológica (izquierda-derecha) de 11 puntos, la religiosidad (religioso/a o no religioso/a), el lugar

Tabla I

Mitos y creencias en función de las categorías identificadas por Bosch y Ferrer (2012)

\begin{tabular}{|l|l|}
\hline Categoría de mitos & Ítems \\
\hline \multirow{2}{*}{ Mitos sobre la marginalidad } & La violencia de género se produce mayoritariamente en las clases sociales más bajas \\
\cline { 2 - 2 } & La violencia de género es un problema que afecta, sobre todo, a los países pobres \\
\hline \multirow{3}{*}{ Mitos sobre los maltratadores } & Cualquier hombre puede perder el control sin ser un maltratador \\
\cline { 2 - 2 } & Los hombres que ejercen violencia de género tienen problemas psicológicos \\
\cline { 2 - 2 } & Un hombre que ha ejercido violencia de género puede ser un buen padre \\
\hline \multirow{3}{*}{ Mitos sobre las mujeres maltratadas } & $\begin{array}{l}\text { Las mujeres que permanecen en relaciones de violencia de género se quedan porque } \\
\text { quieren }\end{array}$ \\
\cline { 2 - 2 } $\begin{array}{l}\text { Mitos que minimizan la importancia de la } \\
\text { violencia de género }\end{array}$ & Las mujeres ejercen tanta violencia en la pareja como los hombres \\
\hline \multirow{3}{*}{ Mitos negacionistas } & Una gran parte de denuncias por violencia de género son falsas \\
\cline { 2 - 2 } & $\begin{array}{l}\text { La legislación en materia de violencia de género debería tratar por igual a mujeres y } \\
\text { hombres }\end{array}$ \\
\hline
\end{tabular}


de residencia (área rural o urbana), el país de origen (España u otros países), la situación económica personal (con dificultades económicas para llegar a fin de mes o sin dificultades), la orientación sexual (heterosexual o no heterosexual) y el estado civil (casado/a o no casado/a).

\section{Análisis de datos}

Con el propósito de dar respuesta al principal objetivo planteado en esta investigación, se llevó a cabo un análisis de clases latentes para identificar subgrupos de personas sobre la base del apoyo a una serie de mitos y creencias sobre la violencia de género. Después de determinar el modelo que mejor se ajustaba a los datos, en función de los Criterios de Información Bayesiana y de Akaike $^{3}$ y la razón de verosimilitud (G2), y tras asignar los casos a las distintas clases identificadas, se estimó un modelo de regresión multinomial con el propósito de predecir la pertenencia a las distintas clases a partir de las experiencias de victimización y las características de las personas encuestadas. En la tabla III se presentan los cocientes de riesgo relativo ( $R R R)$ que hacen referencia a la probabilidad de pertenencia a una clase específica en comparación con la clase de referencia (Zhang y Yu 1998). Para finalizar, se estimó un modelo de regresión lineal múltiple prediciendo las creencias sexistas a partir de la pertenencia a las distintas clases identificadas, las experiencias de victimización propias y vicarias y las características personales de las y los encuestados. Los factores de inflación de la varianza estuvieron dentro de los límites apropiados, sugiriendo que no existen problemas de multicolinealidad en los modelos. Debido a que algunas de las variables presentaron datos perdidos, se aplicaron procedimientos de imputación múltiple mediante ecuaciones encadenadas (MICE). El porcentaje de datos perdidos fue bajo, siendo las únicas variables que los presentaron: conocer a agresor(es) de violencia de género (13,0 \%), conocer a víctima(s) de violencia de género $(11,9 \%)$, ideología política $(9,1 \%)$, religiosidad $(5,6 \%)$, haber sufrido violencia emocional y conductas de control $(3,5 \%)$, haber sufrido violencia física y sexual $(3,1 \%)$ y orientación sexual $(2,2 \%)$. Los coeficientes de regresión y los intervalos de confianza se estimaron y promediaron en los 20 conjuntos de datos generados utilizando técnicas de imputación múltiple.

\section{Resultados}

\section{Clases latentes basadas en la adhesión a los mitos sobre la violencia de género}

En la tabla II se recoge el resumen de los modelos de clases latentes estimados. Como se muestra, se estimaron modelos con un número creciente de clases (1-9) con el propósito de lograr el modelo más parsimonioso que ofreciera un mejor ajuste a los datos. Los nueve modelos estimados fueron comparados en función de tres medidas que consideran la bondad de ajuste y la parsimonia: el Criterio de Información Bayesiana (BIC), el Criterio de Información de Akaike (AIC) y la razón de verosimilitud (G2). Valores más bajos de BIC y AIC y valores más altos de G2 indican un mejor ajuste de los modelos a los datos. De las nueve clases estimadas, el modelo de tres clases obtuvo el valor BIC más bajo (véase la tabla II), indicando ser el modelo más parsimonioso. Asimismo, la solución de tres clases mostró un ajuste aceptable a los datos $\left(X^{2}=502,78 ; p>, 05\right)$.

\section{Tabla II}

Indicadores de bondad de ajuste de los nueve modelos de clases latentes analizados

\begin{tabular}{|l|c|c|c|}
\hline & BIC & AIC & G2 \\
\hline 1 Clase & 8557,99 & 8508,85 & $-4244,42$ \\
\hline $\mathbf{2}$ Clases & 8106,70 & 8003,49 & $-3980,74$ \\
\hline $\mathbf{3}$ Clases & $\mathbf{8 0 8 7 , 6 1}$ & $\mathbf{7 9 3 0 , 3 4}$ & $-3933,17$ \\
\hline 4 Clases & 8098,156 & 7891,74 & $-3903,87$ \\
\hline 5 Clases & 8149,22 & 7883,83 & $-3887,91$ \\
\hline 6 Clases & 8188,92 & 7869,46 & $-3869,73$ \\
\hline 7 Clases & 8245,54 & 7876,94 & $-3863,47$ \\
\hline 8 Clases & 8298,93 & 7871,35 & $-3848,67$ \\
\hline 9 Clases & 8201,78 & 7833,17 & $-3841,59$ \\
\hline
\end{tabular}

Nota: Se imprime en negrita el modelo escogido. $\mathrm{BIC}=$ Criterio de Información Bayesiana;

AIC = Criterio de Información de Akaike; G2 = Razón de verosimilitud.

Como se ilustra en la figura 1 , los perfiles obtenidos para las tres clases identificadas pueden distinguirse con facilidad entre sí. La clase 1 fue la más numerosa, al incluir al 65,8 \% de la muestra $(n=663)$. Este grupo se caracterizó por presentar probabilidades bajas de mostrarse de acuerdo con todos los indicadores que compusieron la escala, a excepción de los ítems "la legislación en materia de violencia de género debería tratar por igual a mujeres y hombres" y "los hombres que ejercen violencia de género tienen problemas psicológicos", donde las personas integradas en este grupo presentaron probabilidades relativamente elevadas de mostrarse de acuerdo con ambas afirmaciones (56,0 \% y $41,1 \%$, respectivamente). Esta clase fue designada como 'adhesión baja a los mitos sobre la violencia de género'.

La clase 2 comprendió al 11,2\% de la muestra ( $n$ =113). Este grupo presentó una probabilidad elevada de mostrarse de acuerdo con los ítems "la legislación en materia de violencia de género debería tratar 
Figura 1

Probabilidades asociadas a cada mito para cada una de las clases latentes identificadas

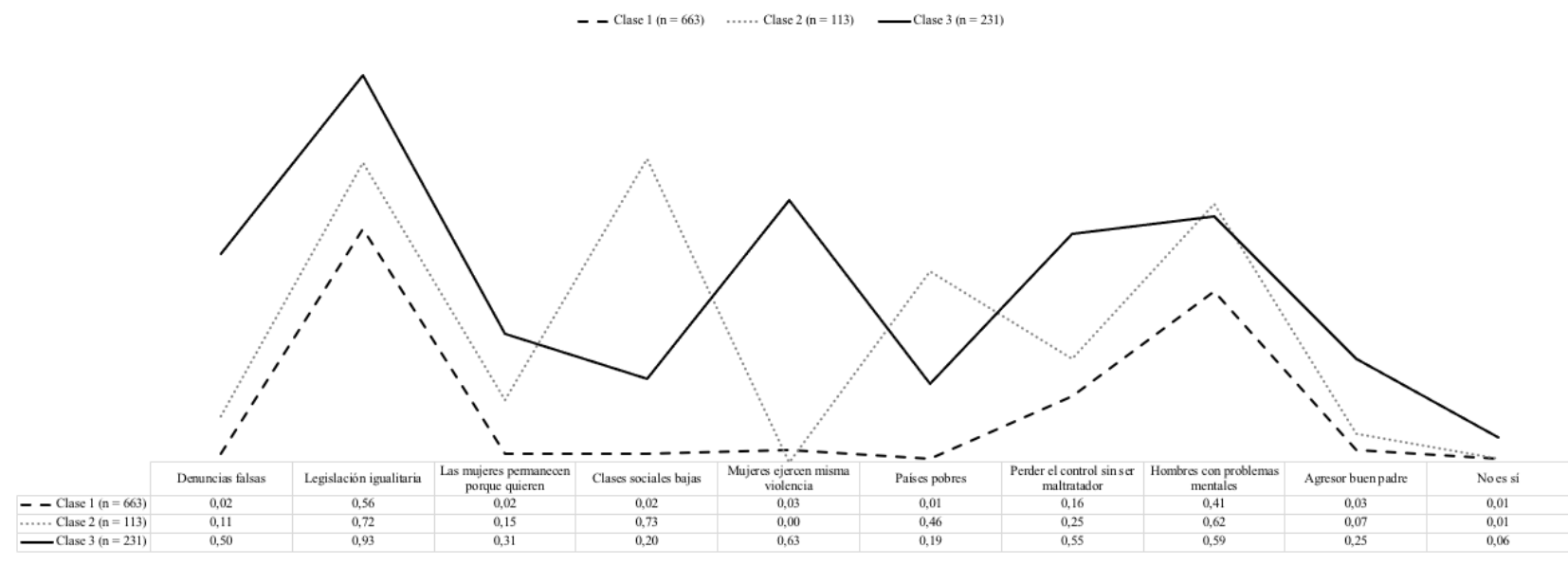

por igual a mujeres y hombres" (72,1\%), "los hombres que ejercen violencia de género tienen problemas psicológicos" (61,8\%), "la violencia de género se produce mayoritariamente en las clases sociales más bajas" $(72,6 \%)$ y "la violencia de género es un problema que afecta, sobre todo, a los países pobres" $(45,7 \%)$. Se considera que esta clase presenta un patrón moderado en la adhesión a los mitos sobre la violencia de género, con énfasis en las creencias sobre la marginalidad del fenómeno.

Por último, la clase 3 estuvo compuesta por el 22,9 $\%$ de la muestra $(n=231)$. Este grupo se caracterizó por la elevada probabilidad de rechazar la legislación actual en materia de violencia de género (92,9\%) y por creer que los maltratadores tienen problemas psicológicos (59,2\%). Asimismo, es probable que las personas de este grupo crean que gran parte de las denuncias por violencia de género son falsas $(50,5 \%)$ y que las mujeres ejercen tanta violencia en las relaciones de pareja como los hombres $(62,9$ $\%$ ). Las personas incluidas en esta clase presentan también probabilidades elevadas de apoyar la creencia de que cualquier hombre puede perder el con- trol sin ser un maltratador (54,8 \%). No obstante, en comparación con la clase 2 , es menos probable que este grupo considere que la violencia de género está vinculada a países o familias pobres (mitos sobre la marginalidad, $19,4 \%$ y $20,3 \%$ respectivamente). A esta clase se la denominó 'adhesión alta a los mitos sobre la violencia de género' y estuvo caracterizada, principalmente, por el respaldo a los mitos que minimizan y niegan la violencia de género.

\section{Características de las clases latentes}

La tabla III recoge las puntuaciones medias y proporciones de las variables independientes para cada una de las clases latentes identificadas. Como se muestra, la media en la escala de sexismo aumentó de forma gradual desde la clase 1 (adhesión baja) hasta la clase 3 (adhesión alta). De manera específica, mientras que la clase 1 puntuó en promedio 2,25 puntos en la escala de sexismo, la puntuación de la clase 2 fue un $7,1 \%$ superior $(M=2,41)$ y el promedio de la clase 3 fue un $16,9 \%$ mayor $(M=$ $2,63)$ que el correspondiente a la clase 1 . Por el con-

Tabla III

Sexismo, victimización propia y vicaria en función de las clases latentes

\begin{tabular}{|l|c|c|c|}
\hline & $\begin{array}{c}\text { Clase 1 }(\mathbf{n}=\mathbf{6 6 3}) \\
\mathbf{M}[\mathbf{9 5} \% \text { IC] }\end{array}$ & $\begin{array}{c}\text { Clase 2 }(\mathbf{n}=\mathbf{1 1 3}) \\
\mathbf{M}[\mathbf{9 5} \% \text { IC }]\end{array}$ & $\begin{array}{c}\text { Clase 3 }(\mathbf{n}=\mathbf{2 3 1}) \\
\mathbf{M}[\mathbf{9 5} \% \mathbf{I C}]\end{array}$ \\
\hline Sexismo & $2,25[2,22-2,28]$ & $2,41[2,35-2,48]$ & $2,63[2,57-2,69]$ \\
\hline Conoce víctima(s) de VG & $\%[95 \%$ IC $]$ & $\%[95 \%$ IC] $]$ \\
\hline Conoce agresor(es) de VG & $46,9 \%[0,43-0,51]$ & $42,4 \%[0,33-0,52]$ & $40,4 \%[0,34-0,48]$ \\
\hline Conductas de control & $31,2 \%[0,28-0,35]$ & $32,3 \%[0,24-0,42]$ & $30,6 \%[0,24-0,38]$ \\
\hline Violencia emocional & $24,2 \%[0,21-0,28]$ & $27,0 \%[0,20-0,36]$ & $36,8 \%[0,30-0,44]$ \\
\hline Violencia física & $22,6 \%[0,20-0,26]$ & $19,8 \%[0,13-0,28]$ & $39,8 \%[0,33-0,47]$ \\
\hline Violencia sexual & $8,8 \%[0,07-0,11]$ & $7,2 \%[0,04-0,14]$ & $18,4 \%[0,14-0,24]$ \\
\hline
\end{tabular}

Nota: Clase 1 = adhesión baja; clase 2 = adhesión moderada; clase 3 = adhesión alta; $M$ = media; $I C$ = intervalo de confianza. 
trario, el porcentaje de personas que indicaron conocer a víctimas de violencia de género descendió de manera gradual desde la clase $1(46,9 \%)$ hasta la clase $3(40,4 \%)$. La clase 2 indicó en mayor proporción conocer a agresores de violencia de género en comparación con las clases 1 y 3 , aunque los porcentajes fueron muy similares (véase la tabla III). En relación con la violencia sufrida en el ámbito de la pareja, la clase 3 (adhesión alta), en comparación con las clases 1 y 2, fue el grupo que indicó en mayor proporción haber sufrido conductas de control, violencia emocional y física. Sin embargo, la proporción de personas que indicaron haber sufrido violencia sexual disminuyó de forma gradual desde la clase 1 $(6,6 \%)$ hasta la clase $3(4,9 \%)$.

Para predecir la pertenencia a las distintas clases identificadas a partir de las experiencias de victimización y las características personales de las y los encuestados se estimó un modelo de regresión multinomial, empleando el grupo de adhesión baja como referencia. Como se muestra en la tabla IV, las personas con niveles superiores de estudios presentaron menor probabilidad de pertenecer a la clase caracterizada por la adhesión moderada, con predominio de mitos sobre la marginalidad (clase 2), en comparación con la clase 1 (adhesión baja de mitos). Asimismo, las personas que se situaron más a la derecha ideológicamente presentaron mayor probabilidad de pertenecer a las clases con mayores niveles de adhesión a los mitos y creencias sobre la violencia de género $(R R R=1,15 ; p<, 001$ para la clase 2; y $R R R=1,26 ; p<, 001$ para la clase 3 ). Por su parte, los hombres tuvieron un incremento del $67,0 \%$ en el riesgo de pertenecer a la clase $3(R R R$ $=1,67 ; p=, 01)$, caracterizada por la adhesión a los mitos que minimizan y niegan la violencia de géne-

Tabla IV

Modelo de regresión multinomial analizando la pertenencia a las clases latentes

\begin{tabular}{|c|c|c|}
\hline \multirow[t]{2}{*}{ Variables } & $\begin{array}{c}\text { Clase 2/1 } \\
\text { Moderada vs. Baja }\end{array}$ & $\begin{array}{c}\text { Clase } 3 / 1 \\
\text { Alta vs. Baja } \\
\end{array}$ \\
\hline & RRR [95 \% IC] & RRR [95 \% IC] \\
\hline Sexo (ref. mujer) & $1,24[0,80-1,90]$ & $1,67[1,16-2,39]^{\star *}$ \\
\hline \multicolumn{3}{|l|}{ Edad (ref. 18-24) } \\
\hline 25-34 & $1,14[0,53-2,44]$ & $1,04[0,50-2,16]$ \\
\hline $35-44$ & $0,85[0,38-1,86]$ & $1,22[0,61-2,47]$ \\
\hline $45-54$ & $0,83[0,37-1,86]$ & $1,07[0,52-2,19]$ \\
\hline $55-65$ & $0,61[0,26-1,47]$ & $0,94[0,45-1,95]$ \\
\hline $66+$ & $0,90[0,37-2,19]$ & $1,17[0,54-2,55]$ \\
\hline \multicolumn{3}{|l|}{ Nivel educativo (ref. primaria o menos) } \\
\hline Secundaria, FP y bachillerato & $0,22[0,10-0,51]^{\star * *}$ & $0,74[0,31-1,76]$ \\
\hline Licenciatura, diplomatura y grado & $0,22[0,09-0,52]^{* * *}$ & $0,42[0,17-1,02]$ \\
\hline Máster y doctorado & $0,30[0,12-0,79]^{*}$ & $0,71[0,27-1,87]$ \\
\hline Hábitat (ref. rural) & $0,77[0,59-1,01]$ & $1,23[0,98-1,54]$ \\
\hline País de origen (ref. otros países) & $1,62[0,47-5,53]$ & $0,63[0,31-1,27]$ \\
\hline Dificultades económicas & $0,74[0,47-1,17]$ & $0,92[0,64-1,32]$ \\
\hline Estado civil (ref. no casado) & $0,97[0,59-1,61]$ & $1,47[0,97-2,23]$ \\
\hline Ideología (izda.-dcha.) & $1,15[1,05-1,26]^{\star \star *}$ & $1,26[1,16-1,36]^{\star \star \star}$ \\
\hline Religiosidad & $1,27[0,79-2,03]$ & $2,17[1,45-3,24]^{\star \star *}$ \\
\hline Orientación sexual (ref. no heterosexual) & $1,41[0,48-4,15]$ & $0,54[0,26-1,13]$ \\
\hline Conoce a víctima(s) de VG & $0,73[0,40-1,31]$ & $0,76[0,47-1,25]$ \\
\hline Conoce a agresor(es) de VG & $1,39[0,76-2,55]$ & $1,15[0,69-1,90]$ \\
\hline Conductas de control & $1,320,75-2,33]$ & $1,32[0,82-2,14]$ \\
\hline Violencia emocional & $0,92[0,48-1,75]$ & $2,11[1,28-3,47]^{\star * *}$ \\
\hline Violencia física & $0,73[0,29-1,85]$ & $1,59[0,87-2,92]$ \\
\hline Violencia sexual & $1,00[0,34-1,67]$ & $0,70[0,32-1,55]$ \\
\hline $\mathrm{F}$ & $3,44^{* \star *}$ & \\
\hline $\mathrm{N}$ & 1.007 & \\
\hline
\end{tabular}

Nota: Grupo de referencia: adhesión baja (clase 1); clase 2 = adhesión moderada; clase 3 = adhesión alta; RRR = cociente de riesgo relativo; IC = intervalo de confianza.

${ }^{*} p<, 05 ;{ }^{* *} p<, 01 ;{ }^{* * *} p<, 001$ 
ro. También aquellas personas que se identificaron como religiosas y que indicaron haber sufrido violencia emocional en sus relaciones de pareja presentaron mayores probabilidades de pertenecer a la clase caracterizada por la adhesión alta de mitos (clase 3), en comparación con la clase 1 (adhesión baja) $(R R R$ $=2,17 ; p<, 001$ y $R R R=2,11 ; p<, 001)$.

\section{Adhesión a mitos y creencias e ideología sexista}

Para finalizar, con el propósito de examinar la relación entre el grado de aceptación de los mitos y creencias sobre la violencia de género y la ideolo-

\section{Tabla V}

Modelo de regresión lineal múltiple analizando las creencias sexistas

\begin{tabular}{|l|c|c|}
\hline Variables & b [95 \% IC] & $\boldsymbol{\beta}$ \\
\hline Clase (ref. bajo) & & \\
\hline Medio & $0,09[0,02-0,15]^{\star \star}$ & 0,06 \\
\hline Alto & $0,22[0,16-0,29]^{\star \star}$ & 0,21 \\
\hline Sexo (ref. mujer) & $0,24[0,19-0,29]^{\star \star}$ & 0,27 \\
\hline Edad (ref. 18-24) & & \\
\hline $25-34$ & $0,10[0,01-0,20]^{\star}$ & 0,08 \\
\hline $35-44$ & $0,11[0,02-0,20]^{\star}$ & 0,10 \\
\hline $45-54$ & $0,12[0,03-0,21]^{\star \star}$ & 0,11 \\
\hline $55-65$ & $0,13[0,03-0,22]^{\star \star}$ & 0,11 \\
\hline $66+$ & $0,19[0,09-0,29]^{\star \star *}$ & 0,14 \\
\hline Nivel educativo (ref. primaria o & & \\
menos) & & \\
\hline Secundaria, FP y bachillerato & $-0,05[-0,18-0,07]$ & $-0,06$ \\
\hline Licenciatura, diplomatura y grado & $-0,11[-0,24-0,01]$ & $-0,13$ \\
\hline Máster y doctorado & $-0,11[-0,25-0,03]$ & $-0,08$ \\
\hline Hábitat (ref. rural) & $0,01[-0,02-0,04]$ & 0,02 \\
\hline País de origen (ref. otros países) & $0,03[-0,08-0,15]$ & 0,02 \\
\hline Dificultades económicas & $-0,03[-0,08-0,02]$ & $-0,04$ \\
\hline Estado civil (casado) & $-0,03[-0,09-0,02]$ & $-0,04$ \\
\hline Ideología (izda.-dcha.) & $0,05[0,04-0,06]^{\star \star *}$ & 0,27 \\
\hline Religiosidad & $0,05[-0,01-0,10]$ & 0,05 \\
\hline Orientación sexual (heterosexual) & $0,14[0,04-0,23]^{\star \star}$ & 0,07 \\
\hline Conoce a víctima(s) de VG & $-0,10[-0,17--0,02]^{\star \star}$ & $-0,11$ \\
\hline Conoce a agresor(es) de VG & $-0,02[-0,06-0,09]$ & 0,02 \\
\hline Conductas de control & $0,05[-0,02-0,11]$ & 0,05 \\
\hline Violencia emocional & $0,02[-0,05-0,09]$ & 0,02 \\
\hline Violencia física & $0,02[-0,08-0,12]$ & 0,01 \\
\hline Violencia sexual & $0,09[-0,02-0,20]$ & 0,05 \\
\hline F & $22,95 * \star$ & \\
\hline R2 Ajustada & 0,33 & \\
\hline N & 1.007 & \\
\hline
\end{tabular}

Nota: $b=$ coeficiente de regresión no estandarizado; $I C=$ intervalo de confianza; $\beta=$ coeficiente de regresión estandarizado. ${ }^{*} p<, 05 ;{ }^{* *} p<, 01 ;{ }^{* *} p<, 001$ gía sexista, se estimó un modelo de regresión lineal múltiple. Como se recoge en la tabla $\mathrm{V}$, los hallazgos muestran que las personas con una adhesión moderada $(b=0,09 ; p=, 011)$ y alta $(b=0,22 ; p<, 001)$ a los mitos sobre la violencia de género puntuaron más alto en la escala de sexismo en comparación con aquellas que secundaron menos mitos. También los hombres $(b=0,24 ; p<, 001)$, las personas mayores de 24 años (véase tabla $V$ ), aquellas que se situaron más a la derecha ideológicamente $(b=0,05 ; p$ $<, 001)$ y quienes se identificaron como heterosexuales $(b=0,14 ; p=, 005)$ presentaron mayores creencias sexistas. Por el contrario, quienes indicaron conocer a víctimas de violencia de género presentaron puntuaciones más bajas en la escala de sexismo $(b$ $=-0,10 ; p=, 008)$. Además de resultar significativa en el modelo, la categoría de mayor adhesión a los mitos y creencias sobre la violencia de género fue el tercer predictor más importante de la ideología sexista $(\beta=0,21)$, por detrás de la ideología política y el sexo (para ambas variables $\beta=0,27$ ). El modelo explicó un porcentaje aceptable de variación en las actitudes sexistas de las personas encuestadas $\left(R^{2}\right.$ ajustada $=0,33$ ).

\section{Discusión Y CONCLUSIONES}

La presente investigación emplea el análisis de clases latentes para estudiar tipologías basadas en la adhesión a una serie de mitos y creencias sobre la violencia de género en España. Asimismo, examina su relación con la victimización en el marco de la pareja y con las creencias sexistas. Los hallazgos suponen una contribución relevante a este ámbito de estudio y tienen implicaciones clave al identificar algunos de los mitos y creencias más extendidos en el imaginario colectivo y el perfil de los grupos hacia los que se podrían orientar principalmente las acciones de sensibilización.

Los resultados ponen de manifiesto que el modelo que mejor se ajusta a los datos es el de tres clases, dando como resultado las categorías de adhesión baja, moderada y alta a los mitos y creencias sobre la violencia de género. La primera clase aglutina la mayor proporción de personas (en torno a dos tercios de la muestra) y se caracteriza por un apoyo generalmente bajo a los ítems que compusieron la escala, a excepción de "la legislación en materia de violencia de género debería tratar por igual a mujeres y hombres" (mito negacionista) y "los hombres que ejercen violencia de género tienen problemas psicológicos" (mito sobre los maltratadores). La clase 2 es la menos numerosa y congrega al $11,2 \%$ de las personas encuestadas. Este grupo se caracteriza por una adhesión moderada a los mitos, destacando el apoyo a los ítems suscritos por la clase 1 , junto con los dos mitos sobre la marginalidad del fenómeno ("la violencia de 
género se produce mayoritariamente en las clases sociales más bajas" y "la violencia de género es un problema que afecta, sobre todo, a los países pobres"). Por último, la clase 3 , caracterizada por un fuerte respaldo a los mitos y creencias sobre la violencia de género, comprende al 22,9 \% de la muestra. Este grupo se caracteriza por apoyar los mitos negacionistas ("una gran parte de las denuncias por violencia de género son falsas" y "la legislación en materia de violencia de género debería tratar por igual a mujeres y hombres") y los que minimizan la importancia de la violencia de género ("las mujeres ejercen tanta violencia en las relaciones de pareja como los hombres"), aunque también los relacionados con los maltratadores ("los hombres que ejercen violencia de género tienen problemas psicológicos" y "cualquier hombre puede perder el control sin ser un maltratador"). En general, las personas integrantes de este grupo muestran una mayor tendencia a suscribir cada uno de los mitos y creencias estudiadas, a excepción de aquellos sobre la marginalidad del fenómeno, que son más acentuados entre los miembros de la clase 2.

Tomados en conjunto, los resultados muestran la elevada presencia de mitos y creencias sobre la violencia de género entre las personas encuestadas. Estos hallazgos revisten especial importancia debido a que estas creencias desvirtúan el fenómeno de la violencia de género, restándole gravedad y situándolo como un hecho puntual fruto de circunstancias extraordinarias (Bosch y Ferrer 2002; 2012). Además, estas creencias responsabilizan a las víctimas (p.ej., mediante el mito del masoquismo, que postula que si las mujeres no abandonan la relación es porque les gusta que las maltraten) y exoneran a los maltratadores (p.ej., apoyando la idea de que los agresores son enfermos mentales, tienen problemas con el alcohol o son celosos) (Peters 2008). A pesar de estos hallazgos, es importante destacar que ninguna de las clases identificadas se caracteriza por apoyar de forma contundente los mitos sobre las mujeres maltratadas (el apoyo osciló entre el 1,9 - 30,9 \% para el ítem "las mujeres que permanecen en relaciones de violencia de género se quedan porque quieren" y fue inferior al 7,0 \% para el ítem "a menudo cuando las mujeres dicen no en realidad quieren decir sí"). Se considera que este hallazgo es relevante, ya que los mitos que conforman esta categoría son los que entrañan mayores repercusiones para las víctimas al responsabilizarlas de la violencia que sufren. Además, los mitos sobre las mujeres maltratadas favorecen que las víctimas no abandonen las relaciones de abuso (Pease y Flood 2008), no denuncien la violencia sufrida y no cuenten con la ayuda que precisan (Grothues y Marmion 2006). En relación con estos hallazgos, se recomienda que futuros estudios examinen la medida en que el patrón de tres clases la- tentes encontrado en el presente trabajo (adhesión baja, moderada y alta) se mantiene y reproduce al emplear otros ítems distintos a los utilizados.

Otra de las principales contribuciones de este trabajo es la identificación de las características que definen a cada una de las clases. A este respecto, los resultados muestran que ciertas variables, como son el sexo, el nivel educativo, la ideología conservadora, la adscripción religiosa y haber sufrido violencia emocional en las relaciones de pareja, son claves para diferenciar entre la adhesión baja, moderada o alta a los mitos y creencias sobre la violencia de género. De manera específica, se halló que las personas con niveles superiores de estudios presentaban menor probabilidad de pertenecer a la clase 2 (adhesión moderada, con predominio de mitos sobre la marginalidad), en comparación con la clase 1 (adhesión baja). También se encontró que ser hombre, situarse más a la derecha ideológicamente, definirse como una persona religiosa y haber sufrido violencia emocional en las relaciones de pareja se asociaba con mayores probabilidades de pertenecer a la clase que apoyó en mayor medida los mitos y creencias sobre la violencia de género. En términos generales, estos hallazgos están en sintonía con la literatura previa (Alfredsson, Ask y Von Borgstede 2016; Waltermaurer 2012) y suponen una contribución relevante a este cuerpo de investigación, por cuanto pueden ser empleados en la orientación de intervenciones futuras, al identificar ciertos grupos como prioritarios en tanto que respaldan en mayor medida los mitos y creencias sobre la violencia de género.

Para finalizar, los resultados de esta investigación muestran la importancia de la adhesión a los mitos y creencias sobre la violencia de género, así como de ciertas variables personales, en la predicción de la ideología sexista. Concretamente, los resultados mostraron que la pertenencia a las clases con mayor adhesión a los mitos sobre la violencia de género, el sexo, la edad, la ideología conservadora y la orientación sexual son variables vinculadas con una mayor presencia de actitudes sexistas. Por el contrario, conocer a víctimas de violencia de género se asoció con una menor puntuación en la escala de sexismo. Estos hallazgos son similares a los encontrados en estudios previos, que han mostrado la relación existente entre el apoyo a los mitos sobre la violencia de género y la ideología sexista (Marques-Fagundes et al. 2015; Megías, Toro-García y Carretero-Dios 2018; Peters 2008). Como muestra de ello, los resultados de la presente investigación son similares a los hallados por Megías y sus colaboradores (2018), quienes encontraron que las personas con mayor adherencia a los mitos sobre la violencia de género eran más sexistas, mostraban creencias más negativas sobre las mujeres que sufren agresiones sexuales, apoyaban en menor medida la ideología feminista y tenían más probabi- 
lidades de responsabilizar a las víctimas y exonerar a los maltratadores en supuestos de violencia de género. También en sintonía con investigaciones previas en el ámbito de las actitudes sexistas (Glick et al. 2002; Kelly, Dubbs y Barlow 2015; Lameiras y Rodríguez 2003), los resultados de este trabajo ponen de manifiesto la relevancia del sexo, la edad, la ideología política y la orientación sexual en este ámbito de estudio. El hallazgo de la orientación sexual resulta novedoso, ya que son escasas las investigaciones que han examinado el efecto de esta variable en la ideología sexista, a pesar de que estudios recientes destacan su importancia como predictor de las actitudes sociales (Schnabel 2018). Además, el único trabajo del que se tiene constancia que ha examinado su relación se ha realizado con población universitaria (León y Aizpurúa 2020).

También son destacables los hallazgos en relación con la victimización en la pareja, por las implicaciones que conllevan. En primer lugar, el hecho de que la proporción de víctimas de violencia emocional, física y conductas de control sea mayor en la clase que suscribe más mitos (véase la tabla III) y que, incluso una vez controlado el efecto de otras variables, las personas que han sufrido violencia emocional tengan mayor probabilidad de pertenecer a la clase que suscribe más mitos, pone de relieve la mayor vulnerabilidad de este grupo, que bien puede respaldar más mitos como resultado de su propia victimización o bien ser victimizado en mayor medida porque, al suscribir más mitos, no identifican ciertas situaciones como violentas. Por su parte, el hallazgo que muestra que conocer a víctimas de violencia de género se relaciona con menores creencias sexistas también tiene implicaciones clave, al poner de relieve el efecto que podría tener la visibilidad de los casos de violencia de género en la reducción de las creencias sexistas. Además, está en sintonía con los encontrados por Vázquez y sus colaboradores (2019), quienes en un estudio llevado a cabo utilizando datos para el conjunto de la Unión Europea, hallaron que la victimización vicaria era más relevante que la propia a la hora de explicar las percepciones en torno a la violencia contra las mujeres.

A pesar de su contribución a este ámbito de estudio, la presente investigación tiene ciertas limitaciones que han de ser abordadas en la interpretación de los resultados. En primer lugar, el diseño transversal del estudio únicamente permite hablar de asociaciones y no de relaciones causales. En consecuencia, puede ser que las creencias sexistas precedan a la adhesión a los mitos sobre la violencia de género, pero también que el apoyo a estos mitos sea el germen de las creencias sexistas. Por otro lado, que la muestra sea no probabilística limita las posibilidades de generalización de los resultados. Sin embargo, es importante señalar que la muestra se extrajo utilizando cuotas de sexo, edad y hábitat y que, al comparar la muestra con los datos demográficos publicados por el Instituto Nacional de Estadística (INE 2018), se encuentra que aquella es bastante parecida a la población española. Por último, es importante indicar que las medidas empleadas para evaluar la violencia en la pareja no permiten distinguir la frecuencia ni la severidad de las distintas formas de violencia estudiadas ni si las mismas se produjeron de manera simultánea o espaciada en el tiempo, como tampoco permiten evaluar si se dieron en relaciones pasadas o actuales. No obstante, se incluyeron indicadores que representan los principales tipos de violencia (conductas de control, violencia emocional, física y sexual) (Naciones Unidas 2014).

A pesar de sus limitaciones, los resultados de esta investigación suponen una importante contribución a este ámbito de estudio al poner de relieve el respaldo social a ciertos mitos y creencias sobre la violencia de género en España. Como se ha advertido, aferrarse a creencias erróneas contribuye a que el ciclo de la violencia continúe y desplaza la responsabilidad de los maltratadores hacia las víctimas (Grothues y Marmion 2006; Policastro y Payne 2013). Además, como señalasen Bosch y Ferrer (2012), el rebrote de los mitos y/o el surgimiento de otros puede no solo dificultar los avances en esta materia, sino también tener repercusiones institucionales (al cuestionar, por ejemplo, los recursos públicos destinados a su prevención y tratamiento) y psicológicas (al alterar la sensación de amenaza en las víctimas potenciales o la atribución de responsabilidad a los maltratadores).

Asimismo, debe destacarse la importancia y la novedad de las evidencias encontradas, ya que han sido muy limitados los estudios empíricos que han analizado los mitos y creencias sobre la violencia de género y su relación con las experiencias de victimización en la pareja y con la ideología sexista. En este sentido, se considera fundamental incidir sobre el hallazgo que muestra que las personas que han sufrido violencia en la pareja presentan mayor adhesión a los mitos y creencias sobre la violencia de género, por las implicaciones que esto conlleva. En primer lugar, porque si las personas que están en relaciones violentas muestran una mayor adhesión a los mitos y creencias sobre la violencia de género, la probabilidad de abandonar estas relaciones podría ser menor. Además, estos hallazgos podrían operar también en etapas previas a la consolidación de las relaciones, pues las personas que presentan mayor adhesión a los mitos podrían ser más vulnerables a establecer relaciones violentas, al tener más dificultades para identificar estas situaciones como tales. Para finalizar, es importante señalar que los hallazgos de esta investigación pueden emplearse desde una perspectiva aplicada en el diseño de iniciativas educativas dirigidas a erradicar mitos y creencias sobre este fenómeno que se encuentran particularmente arraigados. 


\section{NOTAS}

[1] El término 'panelistas' se emplea para hacer referencia a las personas que se inscriben en un panel para realizar encuestas a cambio de recibir incentivos.

[2] Tendencia a responder de forma afirmativa con independencia del contenido de los ítems.

[3] Teniendo en cuenta los estudios basados en simulaciones que indican la tendencia del AIC a sobreestimar el número de clases (Nylun, Asparouhov y Muthén 2007), priorizamos el BIC sobre el AIC en la decisión sobre el número apropiado de clases.

\section{Biblografía}

Alfredsson, H., K. Ask y C. Von Borgstede. 2016. "Beliefs about intimate partner violence: A survey of the Swedish general public". Scandinavian Journal of Psychology 57: 57-64. Doi: 10.1111/sjop.12254.

Ansara, D. L. y M. J. Hindin. 2010. "Psychosocial consequences of intimate partner violence for women and men in Canada". Journal of Interpersonal Violence 26: 16281645. Doi: 10.1177/0886260510370600.

Bohner, G., F. Eyssel, A. Pina, F. Siebler, y T. Viki. 2009. "Rape myth acceptance: Cognitive, affective, and behavioural effects of beliefs that blame the victim and exonerate the perpetrator". $\mathrm{Pp}$. $17-45$ en Rape: Challenging contemporary thinking, editado por M. A. H. Horvath y J. M. Brown. Cullompton: Willan Publishing.

Bosch, E. y V. A. Ferrer. 2002. La voz de las invisibles. Las víctimas de un mal amor que mata. Madrid: Editorial Cátedra. Colección Feminismos.

Bosch, E. y V. A. Ferrer. 2012. "Nuevo mapa de los mitos sobre la violencia de género en el siglo XXI". Psicothema 24: 548-554

Bosch, E., V. A. Ferrer, V. Ferreiro y C. Navarro. 2013. La violencia contra las mujeres: El amor como coartada. Barcelona: Anthropos Editorial.

Briere, J. 1987. "Predicting self-reported likelihood of battering: Attitudes and childhood experiences". The Journal of Research in Personality 21: 61-69. Doi: 10.1016/00926566(87)90026-2.

Centro de Investigaciones Sociológicas (CIS). 2012. "Percepción social de la violencia de género". Consulta 9 de Octubre 2019 (http://www.cis.es/cis/export/sites/default/-Archivos/ Marginales/2960_2979/2968/es2968mar.pdf.)

Centro de Investigaciones Sociológicas (CIS). 2017. "Percepción social de la violencia sexual". Consulta 9 de Octubre 2019 (http://www.cis.es/cis/export/sites/default/-Archivos/ Marginales/3180_3199/3182/es3182mar.pdf.)

Coker, A. L., P. H. Smith, L. Bethea, M. R. King y R. E. McKeown. 2000. "Physical health consequences of physical and psychological intimate partner violence". Archives of Family Medicine 9: 451-457. Doi: 10.1001/ archfami.9.5.451.

Comisión Europea 2016. "Special Eurobarometer 449: Gender-based violence". Consulta 9 de Octubre 2019 (https://data.europa.eu/euodp/data/dataset/ S2115_85_3_449_ENG.)

Copp, J. E., P. C. Giordano, M. A. Longmore y W. D. Manning. 2016. "The development of attitudes toward intimate partner violence: An examination of key correlates among a sample of young adults. Journal of Interpersonal Violence 34: 1357-1387. Doi: $10.1177 / 0886260516651311$

\section{Agradecimientos}

Este trabajo ha sido financiado por el Departamento de Derecho Público y de la Empresa de la Universidad de Castilla-La Mancha.

Dalal, K., M. S. Lee y M. Gifford. 2012. "Male adolescents' attitudes toward wife beating: A multi-country study in South Asia". Journal of Adolescent Health, 50: 437442. Doi: 10.1016/j.jadohealth.2011.09.012.

Dardis, C. M., K. M. Edwards, E. L. Kelley y C. A. Gidycz. 2017. "Perceptions of dating violence and associated correlates: A study of college young adults". Journal of Interpersonal Violence 32: 3245-3271. Doi: $10.1177 / 0886260515597439$.

Delegación del Gobierno contra la Violencia de Género. 2020. "Macroencuesta de Violencia contra la Mujer 2019. Consulta 23 de Diciembre 2020 (https:// violenciagenero.igualdad.gob.es/violenciaEnCifras/ macroencuesta2015/pdf/Macroencuesta_2019_ estudio_investigacion.pdf)

Domenech del Río, I. y E. Sirvent García del Valle. 2016. "The consequences of intimate partner violence on health: A further disaggregation of psychological violence Evidence from Spain". Violence Against Women 23: 1771-1789. Doi: 10.1177/1077801216671220.

Edwards, K. M. 2014. "Intimate partner violence and the rural-urban-suburban divide: Myth or reality? A critical review of the literature". Trauma, Violence \& Abuse 16: 359-373. Doi: $10.1177 / 1524838014557289$.

Ekehammar, B., N. Akrami y T. Araya. 2000. "Development and validation of Swedish classical and modern sexism scales". Scandinavian Journal of Psychology 41: 307314. Doi: $10.1111 / 1467-9450.00203$.

Ferrer, V. A. y E. Bosch. 2014. "Gender violence as a social problem in Spain: Attitudes and acceptability". Sex Roles, 70: 506-521. Doi: 10.1007/s11199-013-0322-z.

Flood, M. y B. Pease. 2009. "Factors influencing attitudes to violence against women". Trauma, Violence \& Abuse 10: 125-142. Doi: 10.1177/1524838009334131.

Glick, P., N. Sakalli-Ugurlu, M. C. Ferreira y M. A. de Souza. 2002. "Ambivalent sexism and attitudes toward wife abuse in Turkey and Brazil". Psychology of Women Quarterly 26: 292-297. Doi:10.1111/1471-6402.t01-100068.

Gracia, E. y J. Herrero. 2006. "Acceptability of domestic violence against women in the European Union: A multilevel analysis". Journal of Epidemiology \& Community Health 60: 123-129. Doi: 10.1136/jech.2005.036533.

Gracia, E., J. Herrero y M. Lila. 2008. "Explaining the immigration-partner violence link: Attitudes towards partner violence among Latin-American immigrants in Spain". The Open Family Studies Journal 1: 31-38. Doi: $10.2174 / 1874922400801010031$.

Gracia, E., M. Lila y F. A. Santirso. 2020. "Attitudes towards intimate partner violence against women in the 
European Union: A systematic review". European Psychologist 25: 104-121. Doi: 10.1027/1016-9040/ a000392

Grothues, C. A. y S. L., Marmion. 2006. "Dismantling the myths about intimate violence against women". Pp. 9-14 en 'Intimate' violence against women: When spouses, partners, or lovers attack, editado por P. K. LundbergLove y S. L. Marmion. Westport, CT, US: Praeger Publishers/Greenwood Publishing Group.

Herrero, J., F. J., Rodríguez y A. Torres. 2017. "Acceptability of partner violence in 51 societies: The role of sexism and attitudes toward violence in social relationships". Violence Against Women 23: 351-367. Doi: 10.1177/1077801216642870.

INE 2018. "España en cifras". Consulta 9 de Octubre 2019. (https://www.ine.es/prodyser/espa_cifras/2018/.)

Kelly, A. J., S. L., Dubbs y F. K. Barlow. 2015. "Social dominance orientation predicts heterosexual men's adverse reactions to romantic rejection". Archives of Sexual Behavior 44: 903-919. Doi: 10.1007/s10508-014-0348-5.

Lameiras, M. y Y. Rodríguez. 2003. "Evaluación del sexismo ambivalente en estudiantes gallegos/as". Revista de Acción Psicológica 2: 131-136. Doi: 10.5944/ ap.2.2.526

León, C. M. y E. Aizpurúa. 2020. “¿Persisten las actitudes sexistas en los estudiantes universitarios? Un análisis de su prevalencia, predictores y diferencias de género". Educación XXI 23: 275-296. Doi: 10.5944/ educXX1.23629

Leung, L. 2019. “Deconstructing the myths about intimate partner violence: A critical discourse analysis of news reporting in Hong Kong". Journal of Interpersonal Violence 34: 2227-2245. Doi: 10.1177/0886260516660298.

Marques-Fagundes, A. L., J. L. Megías, D. M. García-García y K. Petkanopoulou. 2015. "Ambivalent sexism and egalitarian ideology in perception of psychological abuse and (in)vulnerability to violence". Revista de Psicología Social 30: 31-59. Doi:10.1080/02134748. 2014.991519.

Megías, J. L., M. Romero-Sánchez, M. Durán, M. Moya y G. Bohner. 2011. "Spanish validation of the Acceptance of Modern Myths about Sexual Aggression Scale (AMMSA)". The Spanish Journal of Psychology 14: 912-925. Doi: 10.5209/rev SJOP.2011.v14.n2.37.

Megías, J. L., V. Toro-García y H. Carretero-Dios. 2018. "The Acceptance of Myths About Intimate Partner Violence Against Women (AMIVAW) Scale: Development and validation in Spanish and English". Psychology of Women Quarterly 42: 44-61. Doi: 10.1177/0361684317742638.

Naciones Unidas. 2014. Guidelines for producing statistics on violence against women. New York: Department of Economic and Social Affairs.

Nabors, E. L., T. L. Dietz y J. L. Jasinski. 2006. "Domestic violence beliefs and perceptions among college students". Violence and Victims 21: 779-795. Doi: 10.1891/0886-6708.21.6.779.

Nylund, K. L., T. Asparouhov y B. O. Muthén. 2007. "Deciding on the number of classes in latent class analysis and growth mixture modeling: A Monte Carlo simulation study". Structural Equation Modeling 14: 535-569. Doi: 10.1080/10705510701575396.

Organización Mundial de la Salud 2017. "Violence Against Women". Consulta 22 de Diciembre de 2020 (https:// www.who.int/reproductivehealth/publications/ violence/9789241564625/en/)
Parker, E. M., A. C. Gielen, R. Castillo, D. W. Webster y N. Glass. 2016. "Intimate partner violence and patterns of safety strategy use among women seeking temporary protective orders: A latent class analysis". Violence Against Women 22: 1663-1681. Doi: 10.1177/1077801216631436.

Pease, B. y M. Flood. 2008. "Rethinking the significance of attitudes in preventing men's violence against women". Australian Journal of Social Issues 43: 547-561. Doi: 10.1002/j.1839-4655.2008.tb00118.x.

Peters, J. 2008. "Measuring myths about domestic violence: Development and initial validation of the domestic violence myth acceptance scale". Journal of Aggression, Maltreatment \& Trauma 16: 1-21. Doi: 10.1080/10926770801917780.

Policastro, C. y B. K. Payne. 2013. "The blameworthy victim: Domestic violence myths and the criminalization of victimhood". JournalofAggression, Maltreatment\&Trauma 22: 329-347. Doi: 10.1080/10926771.2013.775985.

Roberts, A. R. 2002. "Myths, facts, and realities regarding battered women and their children: An overview". Pp. 3-22 en Handbook of domestic violence intervention strategies. Policies, programs, and legal remedies, editado por A. R. Roberts. New York: Oxford University Press, Inc.

Saint-Eloy, H., J. F. Pittman, G. S. Pettit, J.E. Lansford, J. E. Bates, K. A. Dodge y A. Holtzworth-Munroe. 2017. "Classes of intimate partner violence from late adolescence to young adulthood". Journal of Interpersonal Violence 1-25. Doi: $10.1177 / 0886260517715601$.

Saunders, D. G., A. B. Lynch, M. Grayson y D. Linz. 1987. "The inventory of beliefs about wife beating: The construction and initial validation of a measure of beliefs and attitudes". Violence and Victims 2: 39-57. Doi: 10.1891/0886-6708.2.1.39.

Schnabel, L. 2018. "Sexual orientation and social attitudes". Socious: Sociological Research for a Dynamic World 4: 1-18. Doi: 10.1177/2378023118769550.

Simon, T. R., M. Anderson, M. P. Thompson, A. E. Crosby, G. Shelley y J. J. Sacks. 2001. "Attitudinal acceptance of intimate partner violence among U.S. adults". Violence and Victims 16: 115-126. Doi: 10.1891/08866708.16.2.115.

Swim, J. K., K. J. Aikin, W. S. Hall y B. A. Hunter. 1995. "Sexism and racism: Old-fashioned and modern prejudices". Journal of Personality and Social Psychology 68: 199214. Ddoi: 10.1037/0022-3514.68.2.199.

Toro, V. 2018. "Mitos sobre la violencia de pareja contra las mujeres: Construcción de una escala para su medida y primeras evidencias de sus funciones cognitivas". Tesis doctoral, Departamento de Psicología, Universidad de Granada, Granada.

Vázquez, D., E. Aizpurua, J. Copp y J. J. Ricarte. 2019. "Perceptions of violence against women in Europe: Assessing individual- and country-level factors". European Journal of Criminology 1-19. Doi: $10.1177 / 1477370819859462$.

Waltermaurer, E. 2012. "Public justification of intimate partner violence: A review of the literature". Trauma, Violence \& Abuse 13: 167-175. Doi: 10.1177/1524838012447699.

Wang, L. 2016. "Factors influencing attitude toward intimate partner violence". Aggression and Violent Behavior 29: 72-78. Doi: 10.1016/j.avb.2016.06.005.

Webster, K., A. Ward, K. Diemer, M. Flood, A. Powell, K. Forster y N. Honey. 2018. "Attitudinal support for violence against women: What a population-level survey of the Australian community can and cannot tell 
us". Australian Journal of Social Issues 54: 1-24. Doi: 10.1002/ajs4.56.

Westbrook, L. 2009. "Information myths and intimate partner violence: Sources, contexts, and consequences". Journal of the American Society for Information Science and Technology 60: 826-836. Doi: 10.1002/asi.21021.

Yamawaki, N., M. Ochoa-Shipp, C. Pulsipher, A. Harlos y S. Swindler. 2012. "Perceptions of domestic violence:
The effects of domestic violence myths, victim's relationship with her abuser, and the decision to return to her abuser". Journal of Interpersonal Violence 27: 3195-3212. Doi: 10.1177/0886260512441253.

Zhang, J. y K. F. Yu. 1998. "What's the relative risk? A method of correcting the odds ratio in cohort studies of common outcomes". Special Communication 280: 1690-1691. Doi: 10.1001/jama.280.19.1690.

CARMEN M. ${ }^{a}$ LEÓN es Profesora Ayudante Doctor en la Facultad de Derecho de la Universidad de Castilla-La Mancha. Sus principales líneas de investigación incluyen las percepciones y actitudes hacia la violencia de género, la victimización criminal y la metodología de encuestas.

EVA AIZPURÚA es investigadora en la oficina central de la Encuesta Social Europea (City, University of London) e investigadora visitante en Trinity College Dublin. Sus principales líneas de investigación incluyen la violencia interpersonal, las condiciones de vida de la población reclusa, las percepciones y actitudes hacia la justicia y la metodología de encuestas. 